\title{
Metastatic nodular pneumonia due to septic embolism: a Staphylococcal infection
}

\author{
Sanjeet Krishna Shrestha ${ }^{\mathrm{a}^{*}}$, Brajendra Srivastava ${ }^{\mathrm{b}}$, Rabindra Khunjeli ${ }^{\mathrm{b}}$, Ushab Rana Mousin ${ }^{\mathrm{b}}$
}

${ }^{a}$ DM resident, Pulmonary, Critical care and Sleep Medicine, National Academy of Medical Sciences, Bir Hospital, Kathmandu, Nepal

${ }^{b}$ Consultant, Department of Respiratory Medicine, Shree Birendra Hospital, Chhauni, Kathmandu, Nepal

\section{DOI Name}

http://dx.doi.org/10.3126/jaim.v3i2.14068

\section{Keywords}

Necrotizing pneumonia, Panton-Valentine

Leukocidin, Staphylococcal pneumonia

\section{Citation}

Sanjeet Krishna Shrestha. Metastatic nodular pneumonia due to septic embolism: a Staphylococcal infection. Journal of Advances in Internal Medicine 2014;03(01):68-71.

\begin{abstract}
III-defined lung nodules mimicking secondary metastasis caused by Staphylococcus aureus though defined, is uncommon. We report a 58 years male with chest pain, dry cough, fever and abscess over the nape of neck. Bilateral nodules in the chest $x$-ray resembled metastasis. Workup for malignancy was negative. Induced sputum grew Staphylococcus aureus resistant to penicillin group. The lesions cavitated and produced air-fluid levels. Computed tomography confirmed the peripheral location, right sided hydropneumothorax and loculated fissural collection. Recovery was favourable but delayed with injectable ceftriaxone and levofloxacin initially followed by oral levofloxacin. Review chest radiograph showed residual cavities with small pneumothorax. Follow-up after three months showed resolution of all cavities and pneumothorax. Literature review revealed both Methicillin sensitive and resistant staphylococcus aureus can cause metastatic nodular pneumonia. Rapidly necrosis occurs if the organisms are capable of producing the toxin: Panton-Valentine Leukocidin. But this tendency is more common with community-acquired methicillin resistant strains.
\end{abstract}

\section{INTRODUCTION}

Nodular or round pneumonia occurs less frequently than lobar or bronchopneumonia. When present it is rapidly progressive and can be fatal. It is commonly sub-pleural in location but can be central. Sub-pleural consolidation occurs due to haematogenous spread of infection i.e. septic embolism. Rapidly enlarging and necrotizing nodular pneumonia is etiologically consistent with Staphylococcal infection; both aureus and non-aureus species. ${ }^{1}$ About 2 to $5 \%$ of all community acquired pneumonia is attributed to Staphylococcus aureus(SA). ${ }^{2}$ Methicillin resistant staphylococcus aureus(MRSA) producing Panton Valentine Leukocidin(PVL) cytotoxin are associated with rapidly necrotizing nature of pneumonia. But Methicillin sensitive Staphylococcus aureus(MSSA) infection can also produce similar features if it produces PVL cytotoxin. ${ }^{3}$ Streptococcus pneumoniae, Klebsiella pneumoniae, Actinomycetes, Nocardia, Aspergillus, Legionella, Q fever, M.tuberculosis, are other pathogens producing similar features. ${ }^{1,4}$

Our patient developed rapidly progressive nodular pneumonia with sputum culture growing SA that necrotized despite antibiotics. It required six weeks of antimicrobials for the adequate response and healing.

\section{CASE REPORT}

Here we have a 58 years old thin built male with history of smoking and treated pulmonary tuberculosis 29 years back. He presented with one spike of fever one week prior to the hospital visit, following which he developed chest pain and dry cough. He had exertional breathlessness with few exacerbations in

\footnotetext{
* Corresponding author

Dr. Sanjeet Krishna Shrestha,

DM resident

Pulmonary, Critical care and Sleep Medicine

National Academy of Medical Sciences, Bir Hospital

Kathmandu, Nepal
} 
the past. He was febrile $\left(99^{\circ} \mathrm{F}\right)$ and breathless on presentation with bilateral crackles in chest and oxygen saturation of $86 \%$ in room air. Chest radiograph showed bilateral inhomogeneous nodular lesions. Blood counts revealed leucocyte count of $11,700 / \mathrm{mm}^{3}$ with $80 \%$ neutrophils, haemoglobin of $10.8 \mathrm{~g} /$ dl. Renal functions and electrolytes were normal except urea of $75 \mathrm{~g} / \mathrm{dl}$. Serological screening for HIV, HBsAg and Anti-HCV were all nonreactive. After initial stabilization, the possibility of metastatic malignancy of lung was considered. Hence, ELISA for Carcinoembryonic antigen (CEA), Prostate Specific Antigen (PSA) and Carbohydrate Antigen 19-9 (CA 19-9) were evaluated to identify the primary malignancy. CEA was below reference range but PSA was $7.1 \mathrm{ng} / \mathrm{ml}$. (reference value $=<4 \mathrm{ng} / \mathrm{ml}$ ) and CA 19-9 was $50.2 \mathrm{IU} / \mathrm{ml}$ (reference range $=<35.0 \mathrm{IU} / \mathrm{ml}$ ). Colonoscopy was normal and abdominal ultrasound showed minimal pelvic collection but no mass lesions. Ceftriaxone and azithromycin were used as initial empirical antibiotics. Gram's and acid fast staining of sputum were negative. Blood and urine culture sent from emergency failed to grow any organisms. But sputum grew Staphylococcus aureus sensitive to quinolones, cephalosporins, aminoglycosides, tetracyclines and carbapenems but resistant to penicillins. Hence, the antibiotics were later changed to levofloxacin and ceftriaxone. History and examination reviewed at this time revealed an indurated lesion over the nape of neck, which turned out to be an abscess. The cultures from the drained specimen also showed Staphylococcus aureus. The nodular infiltrates cavitated on the review X-ray done after one week. Contrast tomography of Chest was done suspecting cavitating malignancy. Multiple thick walled cavities of varying sizes were noted in bilateral lung field, predominantly in periphery and upper lobes. Few of these showed air fluid level. Right sided hydropneumothorax with loculated fissural collection was also seen. Incidentally CT also picked up isodense peripheral nodular enhancement in right parasternal region extending from $\mathrm{C} 7$ to T6. There were multiple sub-centimetric lymphnodes around the carina. Possibilty of septic emboli was considered and patient was continued on two weeks of injectable antibiotics: ceftriaxone and levofloxacin. Orally levofloxacin was continued for next four weeks. Chest radiograph at the end of 1 month of therapy showed reduction of size and number of lesions. Then the patient was followed up after 3 months and the CXR only showed emphysematous changes without any nodules or cavities.

\section{DISCUSSION}

Minority of bacterial pneumonia do not produce predictable history and fails to follow the usual natural course. Necrotizing pneumonia is a form of complicated pneumonia which produces multiple areas of consolidation with rapid necrosis. ${ }^{5}$ Such phenomenon can also occur in nodular pneumonia but is uncommon. $^{1}$

Haematogenous and local spread of infection producing metastatic focus is a known complication of Staphylococcal infection. Such complications vary in frequency from 16 to $36 \%$. In a prospectively matched case control study published in 2012, $73 \%$ (84 out of 115) patients with gram positive infection had metastatic septic foci and $19 \%$ of the metastases were in lungs (16 out of 84 ) in the form of lung abscess. ${ }^{6}$ In a recent study published in 2012, metastatic foci of Staphylococcal infection were considerably higher in outpatients (67.2\%) as compared to inpatients (32.8\%). The metastases were most common in the lung (39 out of 67) and the Methicillin sensitive strains were common in the out patients. $^{7}$

The non-detection of a clinically silent focus of infection is a vital factor during clinical management of metastatic gram positive infections. The other important risk factors for metastases of Staphylococcal and other Gram positive infections are delay in treatment, noncompliance with recommended treatment, patient coming directly from the community, positivity of blood culture after 48 hours of admission and persistent fever after 72 hours of admission and treatment. The regular risk factors predisposing to infection also play crucial role in such phenomenon like alcoholism, old age, diabetes mellitus, malignancy and immune-suppression. Our patient came from community after seven days of initial symptoms with a clinically silent abscess over the nape of the neck detected 10 days after admission. ${ }^{6,8-11}$ Septic pulmonary embolism(SPE) like in our case, almost always has a predisposing infective focus prior to the development of pulmonary features. Racheal J. Cook et al. retrospectively analysed 14 cases of SPE and all patients had extra-pulmonary foci of infection prior to development of SPE. ${ }^{12}$

Initial radiograph of our patient taken at emergency room was suggestive of multiple bilateral lung metastases of neoplastic origin. The necrosis of all the nodular opacities further hinted towards necrotizing malignancy. Only after the detection of the neck abscess the diagnosis metastatic necrotizing nodular pneumonia was considered in this case. Bilateral nodular or round pneumonia are relatively uncommon and undiagnosed in adults as compared to children. ${ }^{13}$ More common presentation is solitary pulmonary nodule. Text books define nodular pneumonia to be rarer than lobar or bronchopneumonia. If the mode of spread is haematogenous the lesions are more likely to be bilateral and sub-pleural. The gram positive organisms are to be suspected in such radiological scenario. And if the lesions necrotize then PVL producing Staphylococcus aureus comes into the differential diagnosis. When the 
peripherally located infective nodules necrotize then pleural effusion, pneumothorax, hydro-pneumothorax and empyema complicates the picture due to the rupture of the sub-pleural focus. ${ }^{1}$ Retrospectively it was seen that all these clinical events did occur in our patient in sequence. However, there are two limitations to our presumption: we were unable to characterize PVL status of the Staphylococcus aureus and blood cultures were negative to confirm bacteraemia.

PVL: the cyto-toxin can be produced by both MSSA and MRSA and is responsible for rapid progression of pneumonia and necrosis with complications. Hence, PVL status of Staphylococcus needs to be verified irrespective of resistance pattern. ${ }^{14} \mathrm{PVL}$ producers should receive prolonged therapy (4 to 6 weeks) with sensitive antibiotic. Empirical therapy should include beta-lactam (penicillin, ceftriaxone) or respiratory quinolone (levofloxacin) depending on susceptibility and allergic profile. ${ }^{15}$ Adding clindamycin, linezolid or rifampicin to beta lactam is known to suppress toxin release in PVL strains. ${ }^{16}$ Infectious Disease Society of America (IDSA) and American Thoracic Society (ATS) guidelines on severe community acquired pneumonia states that CA-MRSA requires vancomycin or linezolid. ${ }^{17}$ However, first line antibiotic in adequate dose and duration is sufficient for sensitive strains, as in our case.

\section{Figures}

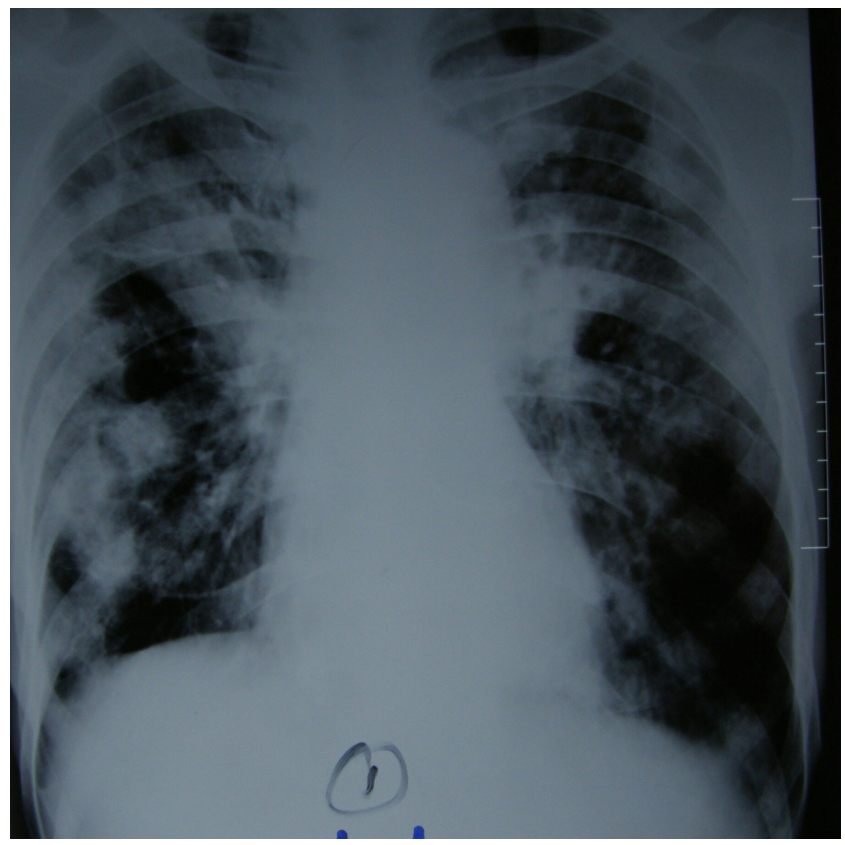

Image 1: Multiple nodules in chest x-ray resembling lung metastasis taken on day one of admission

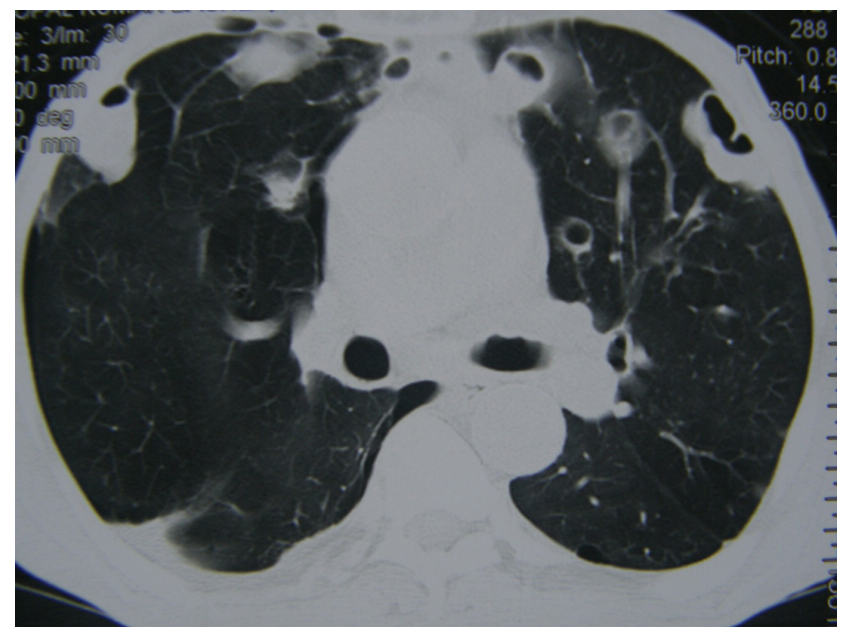

Image 2: CECT chest showing multiple peripherally located cavities with minimal air fluid levels.

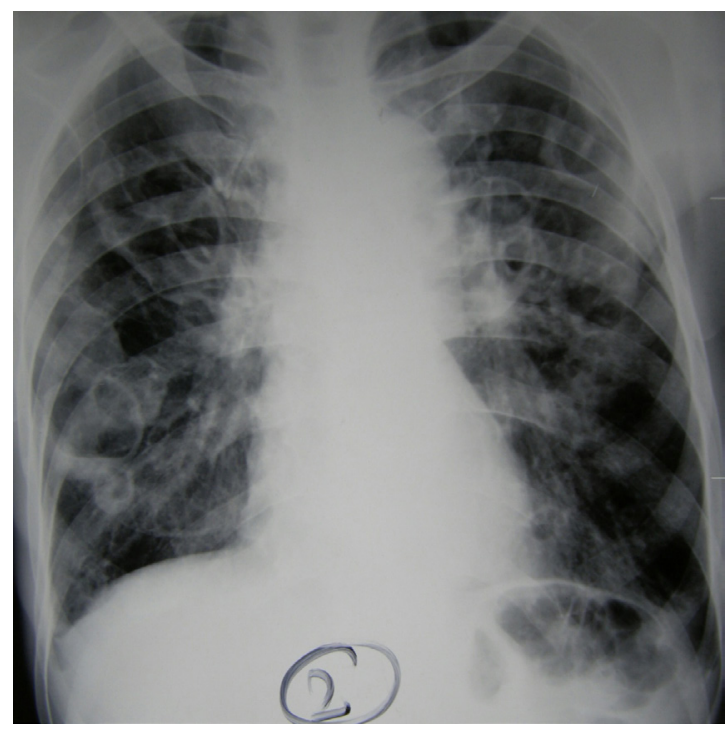

Image 3: Nodules converting into cavities on second week of admission with air fluid levels. 


\section{REFERENCES}

1. Greene RE. The radiology of pulmonary infection. In: Fishman AP, Elias JA, Grippi MA, Senior Rm, Pack AI, editors. Fishman's pulmonary diseases and disorders. 4th ed. Vol 2. New York: McGraw Hill Medical Inc; 2008. p. 2022-4.

2. Valor F, Chebib N, Gillet $Y$, Reix P, Laurent F, Chdiac C, et al. Staphylococcus aurues bronchopulmonary infections: revue de peumologoe Clinique. 2013;69:368-82.

3. Marco T, Monaco M, Vimercati $M$, Ceraminiello $A$, Annalisa P. Methicillin susceptible staphylococcus aurues in skin and soft tissue infections, Northern Italy. Emerg Infect Dis. 2009 Feb;15(2):250-7.

4. Tsai $\mathrm{YF}, \mathrm{Ku} \mathrm{YH}$, Necrotizing pneumonia: a rare complication of pneumonia requiring special consideration. Curr Opin Pulm Med. 2012;18:246-52.

5. Torres A, Ewig S, Mandell L, Woodhead M. editors. Empyema, lung abscess and necrotizing pneumonia. In Chapman SJ, Lee YCG, Davies RJ. Respiratory infections. 1st ed. London: Edward Arnold; 2006. p.385-97.

6. Vos FJ, Kullberg BJ, Sturm PD, Krabbe PF, van Dijk AP, Wanten GA, et al. Metastatic Infectious Disease and Clinical Outcome in Staphylococcus aureus and Streptococcus species Bacteremia. Medicine (Baltimore) 2012 Mar;91(2):86-94.

7. Lukovac E, Koluder-Cimic N, Hadzovic-Cengic M, Baljic, $R$, Ahmetspahic-Begic A. Staphylococcal sepsis and frequency of metastatic foci. Med Arh. 2012;66(3 suppl 1):48-50.

8. Fowler VG Jr, Sanders LL, Sexton DJ, et al. Outcome of Staphylococcus aureus bacteraemia according to compliance with recommendations of infectious diseases specialists: experience with 244 patients. Clin Infect Dis.1998;27:478-86.

9. Fowler VG Jr, Olsen MK, Corey GR, et al. Clinical identifiers of complicated Staphylococcus aureus bacteremia. Arch
Intern Med. 2003;163:2066-72.

10. Lesens $O$, Hansmann $Y$, Brannigan $E$, et al. Positive surveillance blood culture is a predictive factor for secondary metastatic infection in patients with Staphylococcus aureus bacteraemia. J Infect. 2004;48:245-52.

11. Lodise TP, McKinnon PS, Swiderski L, et al. Outcomes analysis of delayed antibiotic treatment for hospitalacquired Staphylococcus aureus bacteremia. Clin Infect Dis. 2003;36:1418-23.

12. Cook RJ, Ashton RW, Aughenbaugh GL, Ryu JH. Septic pulmonary embolism: presenting features and clinical course of 14 patients. Chest. 2005 Jul;128(1):162-6.

13. Cunha BA, Gran A, Simon J. Round pneumonia in a 50 year old man. Respir Care. 2013 Jul;58(7):e80-e2.

14. Royal college of Nursing [Internet]. Panton-Valentin Leukocidin positive Staphylococcal aureus: RCN guidelines for health professionals. [Updated 2011; cited 2014 Sep 12]. Available from: http://www. plymouth.gov.uk/pvl_for_health_professionals.pdf

15. Dumitrescu O, Badiou C, Bes M, Reverdy ME, Vandenesch F, Etienne J, et al. Effect of antibiotics, alone and in combination, on Panton Valentine leukocidin production by a Staphylococcus aureus reference strain. Clin Microbiol Infect. 2008 Apr;14(4):384-8.

16. Stevens DL, Ma Y, Salmi DB, McIndoo E, Wallace RJ, Bryant AE. Impact of antibiotics on expression of virulence-associated exotoxin genes in methicillinsensitive and methicillin-resistant Staphylococcus aureus. J Infect Dis. 2007 Jan 15;195(2):202-11.

17. Mandell LA, Wunderink RG, Anzueto A, Bartlett JG, Campbell GD, Dean NC, et al. Infectious Diseases Society of America / American Thoracic Society consensus guidelines on the management of community-acquired pneumonia in adults. Clin Infect Dis. 2007 Mar 1;44 Suppl 2:S27-S72. 